\title{
Handlungsoptionen von Gewerkschaften und Betriebsräten in multinationalen Unternehmen: Das Beispiel der Niederlande
}

Technologische Entwicklungen, die Liberalisierung des Welthandels und die internationale Kapitalmobilität haben es multinationalen Unternehmen ermöglicht, grenzüberschreitend zu agieren. Im Raum steht die Prognose, derartige Prozesse der Globalisierung führten letztlich zur Angleichung nationaler Produktions- und Wirtschaftsbeziehungen. Basierend auf Fallstudien multinationaler Unternehmen stellt der Beitrag Handlungsoptionen von Betriebsräten und Gewerkschaften vor. ${ }^{1}$

\section{Einleitung}

Die Entscheidungen der Vorstände multinationaler Unternehmen wirken zunehmend über die Grenzen des Nationalstaats hinaus, während die Nationalstaaten im Hinblick auf Steuern und Regulierung miteinander im Wettbewerb um das günstigste Wirtschaftsklima stehen. Darüber hinaus führt die komplexe internationale Organisationsweise vieler dieser multinationalen Unternehmen zu mangelnder Transparenz hinsichtlich des Personalmanagements. Es ist unklar, wie die Aktivitäten der multinationalen Konzerne beim Personaleinsatz und bei der betrieblichen Sozialpolitik gesteuert, überwacht oder koordiniert werden. Im nationalen Umfeld wird diese Kontrollfunktion vom Staat, der Rechtsprechung und von Interessengruppen wahrgenommen, im internationalen Kontext gibt es aber keine vergleichbaren Steuerungsund Überwachungsmechanismen.

Ein Aspekt der andauernden Globalisierungsdebatte betrifft die Frage, ob die Globalisierung zu einer Angleichung nationaler Produktions- oder Wirtschaftssysteme führt oder nicht. Studien kommen hier zu unterschiedlichen Ergebnissen (Berger 1996; Saka 2002).

Jedoch wird die zunehmend verflochtene Weltwirtschaft oft mit der Verbreitung von Elementen eines anglo-amerikanischen Wirtschaftssystems in Verbindung gebracht, das den Freihandel und das Fehlen nationaler Regulierung in den Vordergrund stellt (Ferner/Quintanilla 2002). Da der wirtschaftliche Erfolg eines Landes entscheidend von den Investitionen privater

\begin{tabular}{|lcc|}
\hline Übersicht 1: Fallauswahl & & \\
\hline Wirtschaftsbereich & niederländisch & nicht-niederländisch \\
\hline Elektronik (Industrie) & Philips & Siemens \\
$\begin{array}{l}\text { Nahrungsmittel-, Einrichtungs- und Pflegeprodukte } \\
\text { (Industrie) }\end{array}$ & Unilever & Sara-Lee \\
Einzelhandel (Dienstleistungen) & Ahold & H\&M \\
Telekommunikation (Dienstleistungen) & KPN & Vodafone \\
\hline & & WSI MITTEILUNGEN \\
Quelle: Zusammenstellung der Autoren. & & WS
\end{tabular}

Unternehmen abhängt, stehen Staatsregierungen und Politiker unter Druck, günstige Bedingungen für diese Unternehmen zu schaffen. Obwohl der Wettbewerb in den Bereichen Steuern und Regulierung nicht so scharf ist wie erwartet, spielen die Interessen von einflussreichen Unternehmen in der Tat eine Rolle (Genschel 2001).

Während die Märkte für Kapital und Güter somit stark internationalisiert worden sind, bleibt der Arbeitsmarkt überwiegend eine nationale Angelegenheit. Außerdem bleiben die Beschäftigung, die Entlohnung, die Arbeitsbedingungen und die sonstige betriebliche Sozialpolitik in nationalen Regeln und Institutionen verankert. Diese Institutionen sind normalerweise nicht völlig eigenständig, sondern oft gegenseitig voneinander abhängig (Hollingsworth/Boyer 1997).

In diesem Artikel widmen wir uns den strategischen Wahlmöglichkeiten, die den Gewerkschaften und den Betriebsräten zur Verfügung stehen, um strategisch auf die weitere Internationalisierung der wirtschaftlichen Tätigkeit zu reagieren (vgl. Kochan et al. 1986; Scharpf 1997). Wir stellen folgende Frage: Wie beeinflusst die Globalisierung die Arbeitsbeziehungen und die betriebliche Sozialpolitik in multinationalen Unternehmen in koordinierten Marktwirtschaften wie den Niederlanden und welche Politikempfehlungen können daraus für die Lohnfindung abgeleitet werden?

Die empirische Basis dieses Artikels sind acht Fallstudien in multinationalen Unternehmen, die zumindest eine Betriebsstätte in den Niederlanden unterhalten. Diese Unternehmen wurden aus vier

\footnotetext{
1 Dieser aktualisierte Artikel basiert auf den Analysen von van der Meer et al. (2004). Wir danken unseren Forschungskollegen und unseren Interviewpartnern für die Zusammenarbeit sowie unseren Kollegen bei der SASE und am WSI für ihre Kommentare zu einer früheren Fassung dieses Textes.
}

Marc van der Meer, Associate Professor am Amsterdam Institute for Advanced Labour Studies (AIAS), Universität Amsterdam. Arbeitsschwerpunkte: Industrielle Beziehungen, Arbeitsmarktforschung, Tarifpolitik. e-mail: M.J.S.M.vanderMeer@uva.nI Rob de Boer, ehemaliger wissenschaftlicher Mitarbeiter am Amsterdam Institute for Advanced Labour Studies (AIAS), Universität Amsterdam. Arbeitsschwerpunkte: Europäischer Sozialdialog, Industrielle Beziehungen, Globalisierung. e-mail: rfhdeboer@lycos.nl

Übersetzung aus dem Englischen von Katja Rietzler 
Branchen ausgewählt, nämlich Elektronik (Industrie), Nahrungsmittel-, Einrichtungsund Pflegeprodukte (Industrie), Einzelhandel (Dienstleistungen) und Telekommunikation (Dienstleistungen). Innerhalb dieser Branchen wurde je ein niederländisches und ein nicht-niederländisches multinationales Unternehmen berücksichtigt (Übersicht 1).

Die empirischen Befunde basieren auf schriftlichen Dokumenten, wie z. B. Jahresberichten, Tarifverträgen, Handbüchern zu Arbeitsbedingungen, Zeitungsartikeln usw. sowie mindestens drei ausführlichen Interviews pro Unternehmen. Diese Interviews waren halbstrukturiert und wurden in der Regel mit dem Gewerkschaftsvertreter - soweit vorhanden - (meist von der FNV $)^{2}$ im Unternehmen, einem Betriebsratsmitglied (üblicherweise dem Vorsitzenden) und einem erfahrenen Direktor für Personalmanagement bzw. Human Resource Management oder soziale Angelegenheiten geführt. Die Daten wurden 2003 erhoben und seither aktualisiert.

\section{Corporate Governance in den Niederlanden}

Die Niederlande können als offene Volkswirtschaft charakterisiert werden. Die Bedeutung des Außenhandels ist traditionell hoch (Van Witteloostuijn 1999).

In den vergangenen Jahren haben wir eine anhaltende Debatte über die Rolle des Aufsichtsrats im niederländischen System der Unternehmensführung (Corporate Governance) verfolgen können. Zur Zeit ernennen sich diese Aufsichtsräte im Grunde genommen selbst, wobei die Betriebsräte und die Aktionäre nur eine marginale Rolle spielen. Im März 2003 wurde eine Kommission eingerichtet, um Empfehlungen für das niederländische System der Corporate Governance zu formulieren. Im Dezember desselben Jahres hat die nach ihrem Vorsitzenden benannte TabaksblatKommission die endgültige Version ihres Niederländischen Corporate-GovernanceKodex vorgestellt. Vor dem Hintergrund einer Reihe von Finanzskandalen (Enron, WorldCom, Ahold) hat die Kommission verschiedene Grundsätze und best practices (jedoch keine verbindlichen Regeln) aufgestellt, die darauf ausgerichtet sind, die Integrität, die Transparenz und die Verant- wortung niederländischer Unternehmen zu stärken. Das wichtigste Element ist das Prinzip „befolgen oder erklären“, das es Unternehmen ermöglicht, bei ihrer Unternehmensführung von den Regelungen des Kodex abzuweichen, wenn sie erläutern, warum und wie sie das tun. Im Allgemeinen definieren die Prinzipien, die der Kodex aufstellt, die Rolle der Aufsichtsratsmitglieder und sie stärken die Position der (Hauptversammlung der) Aktionäre. Somit bestätigen sie anscheinend die Tendenz, anglo-amerikanische Elemente ins Niederländische System der Corporate Governance zu integrieren. Die Selbstverpflichtung der niederländischen Regierung, diesen Kodex in der Gesetzgebung zu verankern, bestätigt dies. Erste Anzeichen für den Einzug internationaler Gepflogenheiten in die niederländische Ökonomie waren die jüngsten feindlichen Übernahmen und die erzwungene Zerschlagung von vormals „rheinischen“ Unternehmen durch aktive Hedgefonds und Konsortien internationaler Firmen (Stork, ABN-Amro).

In einem vor Kurzem veröffentlichten Forschungsbericht plädieren der Präsident des Dutch Bureau for Economic Planning und ein weiterer führender Ökonom ausdrücklich für ein „Aktionärsmodell“ der Unternehmensführung, da ihrer Ansicht nach die ,unklaren“ rheinischen Unternehmensgrundsätze keinen wirklichen Schutz der Arbeitnehmer gewährleisteten. Darüber hinaus realisierten ebendiese Beschäftigten einen Lohn oberhalb des markträumenden Lohnes, was zu steigenden Kapitalkosten führe und sich zulasten des Arbeitsplatzaufbaus in der Gesamtwirtschaft auswirke. Schließlich, und das sei „am wichtigsten “, besäßen die Anteilseigner die besten Informationen, um die Risiken der Unternehmung zu verringern, indem sie solche unternehmensspezifischen Risiken über den Kapitalmarkt streuten, was die Autoren als eine effiziente Form der sozialen Sicherung und als "neue“ Stufe im Emanzipationsprozess der Arbeitnehmer ansehen (Bovenberg/Teulings 2008).

\section{Shareholder Value, Restrukturierung und Personalmanagement}

Auf der Grundlage unserer empirischen Untersuchung sind wir zu dem Schluss ge- langt, dass sich in den multinationalen Unternehmen - unabhängig von ihrem Ursprungsland - drei wichtige Veränderungen vollziehen:

- eine Veränderung ihrer strategischen Ziele mit einem Schwerpunkt auf dem Anteilswert (Shareholder Value);

- eine Veränderung ihrer internen Struktur infolge der Einführung von Geschäftseinheiten;

- eine aufkommende explizite Produktivitätsorientierung von Personalmanagementkonzepten.

\subsection{STRATEGISCHE ZIELSETZUNG}

Die meisten der untersuchten Unternehmen haben eine Phase der strategischen Neuorientierung hinter sich. Das amerikanische Unternehmen Sara Lee hat 1978 die traditionsreiche niederländische Kaffeefirma Douwe Egberts gekauft; Philips hat im vergangenen Jahrzehnt verschiedene Prozesse der strategischen Neuorientierung durchlaufen (Operation Centurion); KPN war im Jahr 2001 fast bankrott; Ahold hat im Jahr 2003 eine größere Krise infolge von Missmanagement durchgemacht; und Unilever musste im Jahr 2007 mehrere Betriebe schließen. Während unserer Forschungsarbeiten nahmen Produktion und Beschäftigung bei Vodafone und bei H\&M stetig zu und die Produktionsstätten von Siemens blieben in den Niederlanden auf Kurs.

Es steht außer Zweifel, das mindestens eines der ganz entscheidenden Merkmale des anglo-amerikanischen Systems in diese Unternehmen Eingang gefunden hat: die Orientierung am Shareholder Value (Van der Meer/Van den Toren 2001; Van Witteloostuijn 1999). Der Shareholder Value wird allgemein als der Wert definiert, den ein Anteilseigner aus seiner Investition in ein Unternehmen erhält. Es setzt sich aus Kursgewinnen, Dividenden, Einkünften aus Rückkaufprogrammen und allen anderen Zahlungen der Unternehmung an einen Anteilseigner zusammen (Definition gemäß www.investorwords.com).

Oft sind diese Formen der Unternehmensführung (Corporate Governance) mit verschiedenen Managementauffassungen

\footnotetext{
Der FNV (Bund Niederländischer Gewerkschaften) ist der größte niederländische Gewerkschaftsverband.
} 
verbunden. Mit der zunehmenden Verbreitung der anglo-amerikanischen Orientierung wurde die Maximierung der Rendite je Aktie zum wichtigsten Ziel der Unternehmung, um so die Investoren zufriedenzustellen. Die Dominanz kurzfristiger Ziele der Gewinnmaximierung zeigt sich auch in unserer Untersuchung. Zum Beispiel stellte der Antritt des neuen Vorstandsvorsitzenden Heinrich von Pierer im Jahr 1992 bei Siemens, einem typischen hierarchisch geführten Unternehmen, einen Wendepunkt in der Unternehmensstrategie dar. Mit dem TOP-Programm (time-optimized process, zeitoptimierter Prozess) wurde die Unternehmenskultur weg von einem engen Fokus auf Ingenieurleistungen hin zu marktfähigen Innovationen und mehr Kundenfreundlichkeit orientiert. Dabei wurde ein starker Schwerpunkt auf die Rentabilität und die Rendite der Anteilseigner gelegt (Löwendahl 1999). Ähnliche Entwicklungen vollzogen sich in allen untersuchten Unternehmen. Die Orientierung am Shareholder Value zeigte sich auch an den verstärkten finanziellen Anreizen für das Management, der Existenz von Zielgrößen für die jährliche Rendite, der Qualität der Informationen, die in Geschäftsberichten zur Verfügung gestellt wurden, sowie der Existenz und Ausstattung von Unternehmensabteilungen, die die Beziehungen zu den Investoren pflegen.

\subsection{UNTERNEHMENSRESTRUKTU- RIERUNG}

Alle Unternehmen richten ihr Augenmerk zunehmend auf ihr Kerngeschäft und legen dabei den Schwerpunkt immer mehr auf die Interessen der Investoren. Da es schwierig ist, das Risikoprofil eines diversifizierten Unternehmens zu ermitteln, und die Investoren es vorziehen, selbst zu entscheiden, wie sie ihr Vermögen auf verschiedene Anlageformen aufteilen, konzentrieren sich die Unternehmen auf ein eindeutiges Profil, um Investoren zu gewinnen (Zugehör 2003). Es kann somit der Fall sein, dass multinationale Unternehmen sich tendenziell eher auf eine wirtschaftliche Aktivität konzentrieren, statt ihre Risiken über verschiedene Geschäftsfelder zu streuen.

Die meisten (multinationalen) Unternehmen folgen in ihrem Aufbau heute nicht mehr dem klassischen fordistischen Produktionsmodell, sondern existieren im Rahmen einer großen Vielfalt an weniger hierarchischen und weniger zentralisierten

\begin{tabular}{llc|}
\hline Übersicht 2: Internationale Unternehmensstruktur \\
\hline & Unternehmensaufbau & $\begin{array}{c}\text { Zahl der Länder, in denen das } \\
\text { Unternehmen tätig ist }\end{array}$ \\
\hline Philips & Geschäftseinheiten & 60 \\
Siemens & Geschäftseinheiten & 190 \\
\hline Unilever & Geschäftseinheiten & 100 \\
Sara Lee/DE & Geschäftseinheiten & 55 \\
\hline KPN & Geschäftseinheiten & 3 \\
Vodafone & Tochterunternehmen in den Ländern & 28 \\
\hline Ahold & Tochterunternehmen in den Ländern & 24 \\
H\&M & Tochterunternehmen in den Ländern & 18 \\
\hline & & WS \\
Quelle: Van der Meer et al. 2004. & & MITTEILUNGEN
\end{tabular}

Organisationsformen und Beziehungen zu anderen Organisationen. Innerhalb der Unternehmen zeigt sich dies in Form eines an Bereichen und Geschäftseinheiten orientierten Aufbaus; zwischen Unternehmen wird eine Reihe von Netzwerk- und Vertragsbeziehungen genutzt (Ruigrok/Van Tulder 1995; Supiot 2001). Mit anderen Worten: Transnationale Organisationsformen ersetzen zunehmend die national orientierte Organisationsstruktur. In vielen Unternehmen liegt die Entscheidungsbefugnis nicht mehr beim Management der nationalen Niederlassungen, sondern in internationalen Geschäftsbereichen.

Tatsächlich setzt die stärkere Orientierung am Shareholder Value die Unternehmensleitung erheblich unter Druck, den Gewinn zu maximieren, ein Ziel, das meist in Form einer mehr oder weniger ständigen Unternehmensrestrukturierung verfolgt wird. Unter so modischen Überschriften wie Umstrukturierung des Geschäftsflusses (Business Process Reengineering (BPR)), einsatzsynchrones Management (Just-in-Time Management (JIT)), schlanke Produktion (Lean Production) oder Kerngeschäft (Core Business) werden zahlreiche Unternehmen permanent umstrukturiert, oft bei gleichzeitigen Massenentlassungen von ,überzähligen“ Arbeitnehmern (Van Witteloostuijn 1999). Die Konzentration auf das Kerngeschäft findet auch in Form der Ausgliederung von Servicetätigkeiten (Sicherheitsdienste, Verpflegung, Gebäudereinigung und Personaldienstleistungen) statt (Salverda et al. 2008).

In unseren Fallstudien zeigte sich, dass fünf von acht Unternehmen grenzüberschreitende Geschäftseinheiten aufgebaut hatten. Die Unternehmen fällen Entscheidungen über Investitionen und Produktion auf internationaler Ebene, während gleichzeitig sozialpolitische Themen innerhalb der verschiedenen nationalen Bezugssysteme behandelt werden. Gewerkschaften und
Betriebsräte können die Angelegenheiten, die auf internationaler Ebene geregelt werden, weder kontrollieren noch beeinflussen, weil es keine internationale Organisation von Arbeitnehmerinteressen gibt, die in der Lage wäre, den Entscheidungen, die auf dieser Ebene gefällt werden, etwas entgegenzusetzen. Daher unterliegt die grenzüberschreitende Entscheidungsfindung im Wesentlichen dem Monopol des Managements. Auf der nationalen Ebene sind die Arbeitsbeziehungen und die Verhandlungen über Arbeitsbedingungen zunehmend dezentralisiert und die Interessen der Arbeitnehmer unterscheiden sich stark, was die Macht der Gewerkschaften untergräbt (Übersicht 2).

\subsection{PERSONALMANAGEMENT}

Die verstärkte Konzentration auf den Shareholder Value geht häufig mit einer Dezentralisierung und einer Differenzierung der Unternehmensstruktur einher, was im Bereich des Personalmanagements zu ähnlichen Tendenzen führt. Bei der Veränderung der Beschäftigtenstruktur gibt es große Unterschiede innerhalb des Unternehmens, aber auch zwischen den Wirtschaftsbereichen. Unternehmen in Wirtschaftsbereichen mit einem traditionell hohen Arbeiteranteil, d.h. in der Industrie, haben einen massiven Anstieg der Produktivität verzeichnet und ihre Produktion in einigen Fällen in Niedriglohnländer verlagert. Bei den niederländischen Niederlassungen von Philips, Siemens, Unilever und Sara Lee/DE hat sich somit der Anteil der Angestellten deutlich erhöht. Diese Entwicklung wird sich höchstwahrscheinlich in der Zukunft fortsetzen. Unternehmen im Einzelhandel, also H\&M und Ahold, hängen bei ihrer Tätigkeit jedoch vollständig vom heimischen Markt ab und beschäftigen daher einen weitaus stabileren Anteil an Arbeitern in den Niederlanden. 


\begin{tabular}{|c|c|c|c|}
\hline \multicolumn{4}{|c|}{ Übersicht 3: Gewerkschaften, Betriebsräte und Tarifverträge } \\
\hline & $\begin{array}{l}\text { Zahl und Art der } \\
\text { Tarifverträge }\end{array}$ & $\begin{array}{c}\text { Zahl der im } \\
\text { Unternehmen aktiven } \\
\text { Gewerkschaften }\end{array}$ & $\begin{array}{c}\text { ungefährer } \\
\text { gewerkschaftlicher } \\
\text { Organisationsgrad } \\
\text { in \% }\end{array}$ \\
\hline Philips & 2 (Haustarifverträge) & 4 (FNV, CNV, Unie, VHP) & $25-30$ \\
\hline Siemens & 1 (Branchentarifvertrag) & $3(\mathrm{FNV}, \mathrm{CNV}, \mathrm{VHP})$ & 10 \\
\hline Unilever & 1 (Haustarifvertrag) & 3 (FNV, CNV, Unie) & 25 \\
\hline Sara Lee / DE & 3 (Haustarifverträge) & 3 (FNV, CNV, Unie) & 30 \\
\hline KPN & 1 (Haustarifvertrag) & 4 (FNV, CNV, BTTP, CMHF) & 47 \\
\hline Vodafone & 1 (Betriebsvereinbarung) & Keine & 3 \\
\hline Ahold & $\begin{array}{l}9 \text { (Branchen- und } \\
\text { Haustarifverträge) }\end{array}$ & 4 (FNV, CNV, Unie, VHP) & $\begin{array}{l}\mathrm{AH}^{11)}: 3 \\
\text { Deli XL: } 20\end{array}$ \\
\hline$H \& M$ & 1 (Branchentarifvertrag) & Keine & 3 \\
\hline \multicolumn{4}{|c|}{$\begin{array}{l}\text { 1) Diese Angaben umfassen nur die Supermärkte und nicht den Vertrieb, } \\
\text { wo der Organisationsgrad erheblich höher ist. }\end{array}$} \\
\hline Quelle: Van $\operatorname{der} M$ & t al. 2004 & & MITTEILUNGEN \\
\hline
\end{tabular}

Ein ähnliches Argument gilt für die Unternehmen in der Telekommunikation, obwohl der Anteil der Arbeiter in diesen Unternehmen viel geringer ist. Da der Anteil der Angestellten steigt, dürfte auch die relative Bedeutung von Angestelltengewerkschaften zunehmen. Die Interessen der Angestellten stimmen nicht immer mit denen der Arbeiter überein. Dies kann zu Konflikten innerhalb von Einheitsgewerkschaften oder zwischen Einheitsgewerkschaften und Angestelltengewerkschaften führen. Zudem dürfte die internationale Organisation von Angestelltengewerkschaften sogar noch schwerer zu realisieren sein als die von Einheitsgewerkschaften, da Erstere im Allgemeinen keine europäischen oder weltweit tätigen „Verbündeten“ haben.

Es ist zu berücksichtigen, dass der gewerkschaftliche Organisationsgrad zwischen den untersuchten Unternehmen und Wirtschaftsbereichen stark variiert. Während er infolge des historisch hohen Arbeiteranteils in der Industrie traditionell hoch ist, weisen Unternehmen in den Dienstleistungsbereichen im Allgemeinen einen deutlich niedrigeren gewerkschaftlichen Organisationsgrad auf. Auch innerhalb der Wirtschaftsbereiche gibt es Unterschiede. Als ehemaliges Staatsunternehmen hat KPN immer noch einen großen Anteil an Gewerkschaftsmitgliedern unter seinen Arbeitnehmern, während der gewerkschaftliche Organisationsgrad bei Vodafone sehr gering ist (Übersicht 3).

Im Bereich des Personalmanagements umfassen die typischen anglo-amerikanischen Elemente individuelle Arbeitsverträge, eine flexible Einstellungs- und Entlassungspolitik, ein leistungsabhängiges Entgelt und das Fehlen von kollektiven Versicherungen und Betriebsrenten. In diesem Kontext manifestiert sich die Orientierung am Shareholder Value und an kurzfristiger Gewinnmaximierung zum einen in Form von leistungsabhängigem Entgelt, das an die Stelle regelmäßiger Tariferhöhungen tritt; und zum anderen auch in Form von Veränderungen der betrieblichen Rentensysteme, wobei versorgungsorientierte System von beitragsorientierten Formen abgelöst werden. Veränderungen der Entgeltund betrieblichen Rentensysteme werden von allen untersuchten Unternehmen in Erwägung gezogen.

Die Einführung neuer Technologien und Managementtechniken setzt das Management und die Gewerkschaften auch unter Druck, ihre Strukturen und Grundsätze der Tarifverhandlungen und der betrieblichen Sozialpolitik zu überdenken und die Regelungen von Tarif- und Einzelverträgen mit den neu entstehenden Präferenzen von Abteilungsleitern und einzelnen Beschäftigten in Einklang zu bringen (Van der Meer/Smit 2000). In der Praxis hat dies gelegentlich zum Entstehen von zunehmend komplexen Systemen geführt mit (branchen- oder landesweiten) Manteltarifverträgen, die durch spezifischere Vereinbarungen auf den unteren Ebenen konkretisiert werden sollen. Diesbezüglich kann man eine allgemeine Verschiebung der kollektiven Lohnfindung von der branchenübergreifenden Ebene zu den einzelnen Branchen, von der Branchen- zur Unternehmensebene und von der Unternehmensebene zur Ebene der Produktionsstätte feststellen, die es den Arbeitgebern ermöglicht, Regelungen zu verhandeln, die zu den besonderen Anforderungen von Unternehmen oder sogar einzelnen Arbeitsplätzen passen. Ein weiterer Faktor, der zu dieser Dezentralisierung beiträgt, ist der allgemeine Rückgang des gewerkschaftlichen Organisationsgrades sowie ein Abnehmen der Solidarität unter den Arbeitnehmern mit anderen Worten: eine zunehmende Vielfalt von Arbeitnehmerinteressen. Dabei ist es nicht die Unternehmensseite, die Druck auf die Arbeitsbeziehungen ausübt. Vielmehr ist dies die Konsequenz von zunehmend unterschiedlichen Arbeitnehmerinteressen als eine treibende Kraft des Dezentralisierungstrends (Katz 1993).

Die Dezentralisierung, die Individualisierung der Beschäftigungsbedingungen und die Fragmentierung der Arbeitnehmerinteressen wirken sich stark auf die Position der Gewerkschaften aus. Betriebsräte wiederum sind eher von den Folgen der Internationalisierung betroffen, da ihre Position im Inland an nationales Recht gebunden ist. Wenn beide Akteure ihren Einfluss in multinationalen Unternehmen aufrechterhalten wollen, dann müssen sie sich an diese sich verändernden Umstände anpassen. Für Gewerkschaften kann dies eine verstärkte Konzentration auf Produktionsstatt Verteilungsfragen mit sich bringen, was bedeutet, dass sie in diesem Bereich die Position des Betriebsrats infrage stellen würden. Für Betriebsräte kann dies bedeuten, dass sie sich stärker auf die Entwicklung eines Europäischen Betriebsrats konzentrieren, um auf internationaler Ebene über eine mögliche Gegenmacht zur Unternehmensleitung zu verfügen.

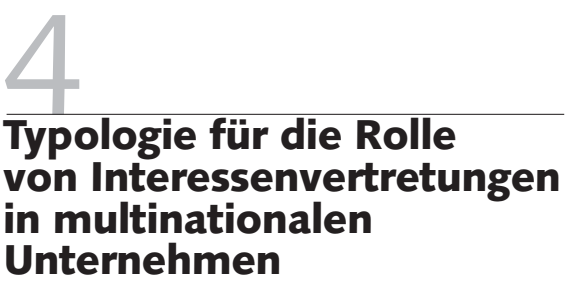

Der Ausgangspunkt für unsere Analyse, wie Arbeitnehmervertretungen auf die Internationalisierung der Produktion reagieren, ist die Verlagerung von Systemen der Koordination zwischen Arbeitgebern und Arbeitnehmern einerseits von der nationalen auf die internationale und andererseits auf die dezentrale Ebene. Es wird angenommen, dass die nationale Koordination von Tarifverhandlungen an Bedeutung verlieren oder gar gänzlich verschwinden wird. Dies wird die traditionelle Arbeitsteilung zwischen Gewerkschaften und Betriebsräten infrage stellen, die davon ausging, dass die Gewerkschaften primär die Arbeitsbedingungen aushandelten, während die Betriebsräte über soziale Belange verhandelten. 
In der Literatur wurden verschiedene Versuche unternommen, sich mit den Veränderungen bei den Arbeitsbeziehungen und bei der Arbeitnehmervertretung auseinanderzusetzen (Boeri et al. 2001; Van het Kaar et al. 2007). Im Zusammenhang mit der Globalisierung unterscheiden wir bei der Entscheidungsfindung zwei Dimensionen, nämlich den Grad der Internationalisierung der Arbeitsbeziehungen und die Art und Weise des Zusammenspiels von Management und Arbeitnehmervertretern. Erstere Dimension bezieht sich darauf, inwieweit internationale Gremien ihre nationalen Entsprechungen bei der Arbeitnehmervertretung ergänzen oder teilweise ersetzen werden. Letztere Dimension betrifft die Rolle der Gewerkschaften. Sie wird entweder als „Monopol“ charakterisiert, wobei die Macht der Gewerkschaften, höhere Löhne und bessere Beschäftigungsbedingungen auszuhandeln, betont wird, oder sie wird als "kollektive Stimme“ beschrieben, wobei die Fähigkeit der Gewerkschaften, die Effizienz interner Arbeitsmärkte zu erhöhen und die Produktivität des Unternehmens zu steigern, im Vordergrund steht (Freeman/Medoff 1984). Eine Gewerkschaft, die die erste Funktion ausübt, konzentriert sich auf Verteilungsfragen, während eine Gewerkschaft, die letztere Rolle erfüllt, auch Produktionsfragen berücksichtigt.

Dies führt zu vier Typen, wobei jeder Quadrant für eine bestimmte Position bei der Interessenvertretung steht. Übersicht 4 zeigt diese Aufteilung und fasst die wesentlichen Kriterien dieser Typologie zusammen. Wie wir in Abschnitt 3 gesehen haben, können die Unternehmenspolitik und die unterschiedliche gewerkschaftliche Organisation das Vorhandensein oder Fehlen einer aktiven Gewerkschaftsvertretung und ihrer relativen Machtposition im Unternehmen erklären. Mit anderen Worten: Historische Umstände und die Institutionen der jeweiligen Volkswirtschaft bestimmen, wie der Shareholder Value Eingang in das Personalmanagement und die Tarifpolitik findet.

\subsection{DEZENTRALISIERTE VERHANDLUNGEN}

Der erste identifizierte Typus (1) geht von einer fortwährenden Dezentralisierung der Verhandlungen über Beschäftigungsbedingungen aus. Die Verhandlungen finden auf den unteren Ebenen des Unternehmens

\begin{tabular}{|c|c|c|c|}
\hline \multicolumn{4}{|c|}{$\begin{array}{l}\text { Übersicht 4: Eine Typologie der zukünftigen Arbeitsbeziehungen } \\
\text { in multinationalen Unternehmen }\end{array}$} \\
\hline \multicolumn{4}{|c|}{ Internationale Ebene } \\
\hline \multirow{2}{*}{$\begin{array}{l}\text { Verhandlung } \\
\text { über } \\
\text { Verteilung }\end{array}$} & $\begin{array}{l}\text { Internationale Koordination (4) } \\
\text { - geringes Vertrauen zwischen } \\
\text { Gewerkschaften und Management } \\
\text { - internationale Organisation von } \\
\text { Gewerkschaften nach Branchen } \\
\text { - keine Rolle für europäische } \\
\text { Betriebsräte außerhalb der EU- } \\
\text { Gesetzgebung } \\
\text { - Arbeitsbedingungen gleichen } \\
\text { sich international an, aber es wird } \\
\text { zwischen Beschäftigtengruppen } \\
\text { unterschieden }\end{array}$ & $\begin{array}{l}\text { Internationale „Unternehmens- } \\
\text { gewerkschaften“ (3) } \\
\text { - Gewerkschaft oder europäischer } \\
\text { Betriebsrat an strategischen Ent- } \\
\text { scheidungen beteiligt } \\
\text { - internationale gewerkschaftliche } \\
\text { Organisation folgt der internen } \\
\text { Organisation des multinationalen } \\
\text { Unternehmens } \\
\text { - Europäischer Betriebsrat spielt } \\
\text { eine koordinierende Rolle bei Tarif- } \\
\text { verhandlungen } \\
\text { - Arbeitsbedingungen gleichen } \\
\text { sich international an, aber es blei- } \\
\text { ben individuelle Unterschiede }\end{array}$ & \multirow{2}{*}{$\begin{array}{l}\text { Verhandlung } \\
\text { über } \\
\text { Produktion }\end{array}$} \\
\hline & $\begin{array}{l}\text { Dezentralisierte Verhandlungen } \\
\text { (1) } \\
\text { - geringes Vertrauen zwischen } \\
\text { Gewerkschaften und Management } \\
\text { - Gewerkschaften nur an Vorteil } \\
\text { für eigene Mitglieder interessiert } \\
\text { (rentseeking) } \\
\text { - Rolle des Betriebsrats orientiert } \\
\text { sich an gesetzlichen Mindest- } \\
\text { standards } \\
\text { - Bei den Arbeitsbedingungen } \\
\text { wird zwischen Beschäftigten- } \\
\text { gruppen unterschieden }\end{array}$ & $\begin{array}{l}\text { Lokale Koalitionen für Produk- } \\
\text { tivität (2) } \\
\text { - Gewerkschaften werden auf der } \\
\text { Betriebsebene akzeptiert } \\
\text { - Gewerkschaften und Betriebs- } \\
\text { räte gewinnen die Belegschaft für } \\
\text { die Unternehmenspolitik } \\
\text { - Abgrenzung zwischen Gewerk- } \\
\text { schaften und Betriebsräten ver- } \\
\text { schwimmt } \\
\text { - Betriebsräte beraten zu Tarif- } \\
\text { verträgen und setzen diese im } \\
\text { Unternehmen um } \\
\text { - Betriebliche Rahmenverein- } \\
\text { barungen mit individuell verein- } \\
\text { barten Arbeitsbedingungen }\end{array}$ & \\
\hline \multicolumn{4}{|c|}{ Dezentrale Ebene } \\
\hline
\end{tabular}

statt (von der Unternehmung über einzelne Geschäftsfelder oder Abteilungen bis hin zu einzelnen Betriebsbereichen). Folglich werden die Einheiten, für die verhandelt wird, kleiner. Diese Entwicklung zeigt sich in den meisten der untersuchten Unternehmen. Das Verhältnis zwischen Gewerkschaften und Management wird zumeist als formal beschrieben, wenngleich leitende Manager und erfahrene Gewerkschaftsvertreter in einigen Unternehmen eine offene und informelle Kommunikation pflegen, da sie sich bereits seit längerer Zeit kennen. Die Hauptstrategie der Gewerkschaften richtet sich darauf, die Beschäftigung zu erhalten, die Entlohnung und die Arbeitsbedingungen für die Beschäftigten zu verbessern, während das Management die Strategie verfolgt, die Gewerkschaften auf Distanz zu halten. Dies kann zu einem von geringen Vertrauen geprägten Verhältnis von Gewerkschaften und Management führen. In Zeiten wachsender Gewinne werden sich die Verhandlungen zwischen Arbeitnehmern und Arbeitgebern auf die Verteilung des erwirtschafteten Mehrwerts konzentrieren, während es in Phasen rückläufiger Beschäftigung zu Arbeitskonflikten oder zu Verhandlungen über Sozialpläne kommen kann.
Dass bei solchen Verhandlungen die Interessenperspektiven von Arbeitern und Angestellten auseinanderdriften können, ist bekannt. Das kann dazu führen, dass für diese Beschäftigtengruppen in verschiedenen Unternehmen getrennte Tarifverträge verhandelt werden, weil die Gewerkschaften gezwungen sind, sich auf die Regelung von Verteilungsfragen für ihre eigenen Mitglieder zu konzentrieren. Eine weitere Dezentralisierung der Verhandlungen kann zur Festlegung „maßgeschneiderter“ Beschäftigungsbedingungen für jeden einzelnen Arbeitnehmer führen, wobei die Rolle der Gewerkschaften darauf beschränkt bleibt, den Ablauf des Entscheidungsprozesses zu bestimmen.

Darüber hinaus gründet sich die Personalpolitik zunehmend auf wirtschaftliche Überlegungen; Entgeltsysteme werden weiterhin die individuelle Leistung als wichtigste Determinante des Gehaltsniveaus in den Vordergrund stellen und andere personalpolitische Maßnahmen werden ebenfalls auf Kostenbegrenzung und Gewinnmaximierung zielen. Dies geschah bei Philips mit der Einführung eines leistungsorientierten Entgelts im Jahr 2000 und bei KPN, wo es durch die Einführung eines persönlichen Kontensystems im Jahr 2007 
$\mathrm{zu}$ einer weiteren Individualisierung der Entgeltpolitik kam.

In diesem Prozess werden die Funktionen von Gewerkschaften und Betriebsräten getrennt, wobei Letztere weiterhin an Verhandlungen über zusätzliche soziale Maßnahmen beteiligt sind und in manchen Fällen in Produktionsangelegenheiten zu Rate gezogen werden, die größtenteils die Angelegenheit der Unternehmensleitung bleiben. Dies ist bei H\&M der Fall, wo die Unternehmung Branchentarifverträgen folgt und die Gewerkschaften im Unternehmen keine wirkliche Funktion haben, während den Betriebsräten eine Rolle im Bereich der betrieblichen Sozialpolitik zugebilligt wird. Bei Philips war die Arbeitsteilung zwischen Gewerkschaften und Betriebsrat immer sehr streng, obwohl die Rolle des Betriebsrats in jüngster Zeit dahingehend verändert wurde, dass er als Rumpfinstitution für Arbeitnehmerinteressen beratend an strategischen Fragen beteiligt wird.

\subsection{PRODUKTIVITÄTSKOALITIONEN}

Dezentralisierung liegt auch der zweiten Konstellation (2) zugrunde, aber sie wirkt sich anders auf die Arbeitsbeziehungen aus. Hier steht die Unternehmensleitung den Gewerkschaften im Unternehmen wohlwollend gegenüber, da sie für die notwendige Unterstützung der Unternehmenspolitik sorgen können. Personalpolitik wird als gemeinsame Sache von Unternehmensleitung und Arbeitnehmervertretern definiert. Dabei müssen die Gewerkschaften nicht über eine hohe Mitgliederzahl verfügen, aber die Unterstützung aller Beschäftigten, der Gewerkschaftsmitglieder wie der Nicht-Mitglieder, ist von zentraler Bedeutung.

Die Gewerkschaften sind an den Verhandlungen zu Haustarifverträgen beteiligt, die nicht nur Verteilungsfragen wie Löhne und Arbeitszeit beinhalten, sondern auch eine Reihe von Produktionsangelegenheiten wie die Organisation der Fertigung, Arbeitszeitmodelle, Training, Personalentwicklung, Beratung und Unterstützung bezüglich individueller Fähigkeiten und Lernprozesse usw. Beispielsweise haben die Haustarifverträge bei Unilever die Form von Rahmenverträgen mit groben Richtlinien, von denen einige vom Betriebsrat dezentral im Unternehmen umgesetzt werden sollen. Zum Teil „spielen“ Gewerkschaften und Betriebsräte „in der
Mannschaft des Managements", um eine möglichst hohe Rentabilität und Wettbewerbsfähigkeit zu erreichen und somit die heimische Beschäftigung im Unternehmen zu schützen (siehe van der Meer 2004). Ein mögliches Merkmal dieses Typs ist außerdem, dass ein Vertrauensverhältnis zwischen Gewerkschaften und Management entsteht, insbesondere in Unternehmen mit einer qualitativ hochwertigen Produktion und spezialisierten Mitarbeitern.

Bei Unilever war dies über einen längeren Zeitraum der Fall, wobei das Unternehmen auch die gewerkschaftliche Betriebspolitik finanziell unterstützt hat, indem u.a. Bildungsmaßnahmen für Gewerkschaftsmitglieder vom Unternehmen finanziert wurden. Als Unilever jedoch im Oktober 2007 eine größere Umstrukturierung, den weltweiten Verlust von 20.000 Arbeitsplätzen und die Schließung von drei Betrieben, einschließlich des 1883 errichteten Calvé-Werks in Delft, ankündigen musste, kühlte die Beziehung zwischen Gewerkschaften und Management gänzlich ab.

Bei Vodafone gibt es keine Gewerkschaftsarbeit im Unternehmen, und die Kompetenzen des Betriebsrats reichen bis zur Verhandlung über primäre Beschäftigungsbedingungen, Betriebsrenten und andere soziale Umstände außerhalb des Einflussbereichs von nationalen Gewerkschaften. Bei schwierigen Entscheidungen über detaillierte Betriebsrentenpläne werden die Betriebsräte von externen Experten und Unternehmensberatern unterstützt. Unseren Informationen nach koordiniert der Betriebsrat seine Aktivitäten nicht auf internationaler Ebene.

\subsection{INTERNATIONALE UNTERNEHMENSGEWERKSCHAFTEN}

Beim dritten Typ (3) wird die Internationalisierung des Entscheidungsprozesses von einer ausgeprägten Internationalisierung der Arbeitnehmervertretungsgremien innerhalb der multinationalen Unternehmen begleitet. Gewerkschaften von verschiedenen Einrichtungen eines multinationalen Unternehmens in verschiedenen Ländern arbeiten grenzüberschreitend zusammen und verhandeln mit der Unternehmensleitung. Die Beziehungen zwischen Arbeitnehmern und Arbeitgebern sind eher durch Konsens als durch Konflikte gekennzeichnet. Die beiden Seiten vertrauen einander und sprechen offen über die jeweiligen Pro- bleme. Das gemeinsame Ziel ist es, den Marktanteil des Unternehmens zu halten oder zu erhöhen, um die Beschäftigung aufrechtzuerhalten oder auszuweiten. Eine wichtige Voraussetzung für eine solche Entwicklung ist die strategische Ausrichtung des Managements. Dieses sieht das Humankapital als zentralen Aktivposten und bemüht sich um die Mitwirkung seiner Belegschaft, um die Produktivität und die Produktion zu steigern. Ein reines Beispiel dieses Typus fand sich in unserer Erhebung nicht. Keines der von uns erfassten Unternehmen hat die Rechte des europäischen Betriebsrats über die Regelungen der europäischen Gesetzgebung hinaus ausgedehnt. Jedoch kann die internationale Unternehmensleitung die Mitarbeiter auch im Bereich der sozialen Verantwortung des Unternehmens konsultieren. Dieses war bei H\&M der Fall.

Für Arbeitnehmervertreter ist es schwierig eine international abgestimmte Position zu erreichen, wenn man berücksichtigt, wie unterschiedlich ihre Möglichkeiten sind, gemeinsame Arbeitnehmerinteressen im Rahmen einer ,internationalen Unternehmensgewerkschaft" zu vertreten, die entsprechend der verschiedenen Geschäftsbereiche des Unternehmens organisiert ist. In diesem Fall werden Tarifverträge zunehmend Fragen der Produktion abdecken und sich weniger auf Verteilungsfragen beschränken. Kahancova (2007) zeigt beispielsweise, dass die Personalpolitik in einem multinationalen Unternehmen zwar internationalisiert wurde, aber die starken Hindernisse für die Zusammenarbeit der (europäischen) Betriebsräte und der nationalen Gewerkschaften zu unterschiedlichen Praktiken in ost- und westeuropäischen Niederlassungen führten. Oder, wie ein Mitglied des Ahold Vorstands jüngst argumentierte: „Niederländische Betriebsräte können nicht erwarten, dass sie in Unternehmen, die $90 \%$ ihres Umsatzes außerhalb der Niederlande verdienen und in denen $85 \%$ der Belegschaft in Abteilungen im Ausland beschäftigt sind, besondere Rechte erwerben “(Vos/Gründemann 2008, S. 317).

\subsection{INTERNATIONALE KOORDINATION}

Der vierte Typ (4) umfasst die Entwicklung eines formalen Verhandlungsmodus auf internationaler Ebene und eine wachsende Rolle für internationale Gewerkschafts- 
organisationen in den verschiedenen Wirtschaftsbereichen. Verhandlungen werden zwischen den verschiedenen nationalen Gewerkschaften eines Wirtschaftsbereichs zunehmend koordiniert. Bei den Tarifverhandlungen geht es in erster Linie um Arbeitsnormen, Entgelt und andere Beschäftigungsbedingungen. Des Weiteren werden die Arbeitsbedingungen sich international angleichen, aber auf dezentraleren Ebenen wird weiterhin zwischen verschiedenen Beschäftigtengruppen differenziert.

Die Rolle der europäischen Betriebsräte wird nicht über die entsprechenden Regelungen der europäischen Gesetzgebung hinaus ausgedehnt. Da sich die Gewerkschaften nach Branchen organisieren, bleibt die Bedeutung von europäischen Betriebsräten, die gemäß der Struktur ihrer Unternehmen organisiert sind, marginal. Insgesamt bleibt die traditionelle Arbeitsteilung zwischen Gewerkschaften und Betriebsräten weitgehend erhalten, während die Verhandlungen internationalisiert werden.

Die Wahrscheinlichkeit dieses Szenarios hängt stark vom Grad der internationalen Solidarität zwischen Gewerkschaften ab, d.h. von der Fähigkeit der Gewerkschaften und ihrer Zusammenschlüsse, sich grenzüberschreitend zu organisieren. Es ist jedoch, wie der teilweise Zusammenbruch der Doorn-Gespräche zwischen den Gewerkschaftsdachverbänden Deutschlands, der Niederlande, Belgiens und Luxemburgs 2003 gezeigt hat, oft schwer für Gewerkschaften, die großen Unterschiede in ihrer Kultur, ihrer Tradition und in ihren Interessen zu überwinden. Offensichtlich hält ein Gefangenendilemma die Gewerkschaf- ten davon ab, viel in die Entwicklung einer internationalen Gewerkschaftsvertretung zu investieren. Wenn sich Gewerkschaften eines Landes international engagieren, dann werden Gewerkschaften in anderen Ländern diesem Beispiel nicht unbedingt folgen, denn die zu erwartende Rendite ist sehr gering.

\section{Schlussfolgerungen}

Bislang sind Gewerkschaften und Betriebsräte in etablierten Unternehmen relativ gut verankert. Ihre Position wird jedoch von drei miteinander verbundenen Faktoren bedroht, die in diesem Artikel behandelt wurden.

(1) Unternehmen definieren ihre strategischen Ziele zunehmend über eine Verbesserung ihres Shareholder Value. Bei Hauptversammlungen spielen Arbeitnehmervertreter keine unmittelbare Rolle. In Ausnahmefällen billigt man dem Betriebsrat höchstens eine beratende Funktion zu.

(2) Multinationale Unternehmen haben ihre internationale Organisation in internationale Geschäftsfelder umstrukturiert, bei denen es keine Gegenmacht in Form von Arbeitnehmervertretern gibt.

(3) Personalpolitik wird in multinationalen Unternehmen zunehmend in Bezug auf individuelle Leistung definiert, wobei Gewerkschaften nur die Abläufe, nicht aber die Inhalte der Arbeitsbedingungen festlegen.
Diese Entwicklungen führen zu einer stärkeren Gewichtung von anglo-amerikanischen Geschäftspraktiken in den Niederlanden und verändern die strategischen Handlungspositionen von Gewerkschaften. Zwei Dimensionen wurden dabei unterschieden, nämlich der Grad der Internationalisierung der Arbeitsbeziehungen und die Art und Weise des Zusammenspiels zwischen Management und Arbeitnehmervertretern.

In einer stark vernetzten Weltwirtschaft und mit Blick auf sinkende Mitgliederzahlen sowie die Zersplitterung von Arbeitnehmerinteressen kann es wichtiger sein, günstige Voraussetzungen für die Wettbewerbsfähigkeit eines inländischen Unternehmens im internationalen Umfeld zu schaffen, als bessere Arbeitsbedingungen auszuhandeln, zumal Letztere in den meisten multinationalen Unternehmen ohnehin überdurchschnittlich gut sind. Die Beteiligung an Diskussionen über Produktivitätsfragen kann die Unterstützung der Beschäftigten für die Unternehmenspolitik in diesem Bereich erhöhen. Produktivitätszuwächse steigern die Wettbewerbsfähigkeit, was wiederum eine stabilere Beschäftigungslage schafft. In dieser Hinsicht werden die Gewerkschaften möglicherweise in einem Bereich aktiv, der traditionell den Betriebsräten vorbehalten war, nämlich die verbindliche Mitwirkung bei strategischen Angelegenheiten. Betriebsräte sollten die Vertretung der Belegschaft in offiziellen Sitzungen durch Formen der direkten Arbeitnehmerbeteiligung im ganzen Unternehmen ergänzen. 
Berger, S. (1996): Introduction, in: Berger, S./Dore, R. (Hrsg.): National Diversity and Global Capitalism, Ithaca

Bovenberg, L./Teulings C. N. (2008): Rhineland Exit, CPB discussion paper 101, Den Haag

Boeri, T./Brugiavini, A./Calmfors, L. (Hrsg.) (2001): The Role of Unions in the Twenty-First Century, Oxford

Commissie Tabaksblat (2003): The Dutch corporate governance code: principles of good corporate governance and best practice provisions, download: http://www.regering.nl/Begrippenlijst/C/Corporate_ governance_code_Tabaksblat

Ferner, A./Quintanilla, J. (2002): Between Globalisation and Capitalist Variety: Multinationals and the International Diffusion of Employment Relations, in: European Journal of Industrial Relations 3, S. 243-250 Freeman, R./Medoff, J. (1984): What Do Unions Do?, New York Genschel, P. (2001): Globalization, Tax Competition and the Fiscal Viability of the Welfare State, MPIfG Working Paper 1

Hall, P./Soskice, D. (Hrsg.) (2001): Varieties of Capitalism. The Institutional Foundations of Comparative Advantage, Oxford

Hollingsworth, J./Boyer, R. (1997): Coordination of Economic Actors and Social Systems of Production, in: Hollingsworth, J./Boyer, R. (Hrsg.): Contemporary Capitalism. The Embeddedness of Institutions, Cambridge Kahancova, M. (2007): Making the most of diversity. Social interaction and variation in employment practices in a multinational company, Amsterdam

Katz, H. C. (1993): The Decentralisation of Collective Bargaining: A Literature Review and Comparative Analysis, in: Industrial and Labour Relations Review 1, S. 3-22

Kochan, T./Katz, H. C./McKersie, R. (1986): The Transformation of American Industrial Relations, Ithaca

Loewendahl, H. (1999): Siemens' "Anglo-Saxon" Strategy: Is Globalising Business Enough?, in: German Politics 1, S. 89-105
Ruigrok, W./Van Tulder, R. (1995): The Logic of International Restructuring, London

Saka, A. (2002): Institutional limits to the internationalisation of worksystems, A comparative study of three Japanese multinational companies in the UK, in: European Journal of Industrial Relations 3, S. 252-275 Salverda, W./Van Klaveren, M./Van der Meer, M. (2008): Low wage work in the Netherlands, New York

Scharpf, F. (1997): Games Real Actors Play - Actor-centered Institutionalism in Policy Research, Boulder

Supiot, A. (2001): Beyond Employment. Changes in Work and the Future of Labour Law in Europe, Oxford

Van der Meer, M. (2004): Die Koordinierte Dezentralisierung der Tarifpolitik in den Niederlanden, in: WSI-Mitteilungen 7, S. 356-362 Van der Meer, M./De Boer, R./Houwing, H./Kahancova, M./Raess, D./Visser, J. (Hrsg.) (2004): The impact of globalisation on industrial relations in multinational corporations in the Netherlands, Amsterdam Van der Meer, M./Smit, E. (2000): Innovatie of imitatie? CAO-vernieuwing op ondernemingsniveau, Elsevier

Van der Meer, M./Van den Toren, J. P. (2001): Wordt de Zeggenschap uitbesteed? De Veranderende Institutionele Posities van Belanghebbenden in het Nederlandse Ondernemingsmodel, in: Tijdschrift voor Arbeidsvraagstukken 1, S. 95-110

Van het Kaar, R./Smit, E./Buitelaar, W./Collard, R./Van der Meer, M./Popma, F./Tros, F. (2007): Vier scenarios voor de toekomst van de medezeggenschap, Delft

Van Witteloostuijn, A. (1999): De Anorexiastrategie. Over de Gevolgen van Saneren, De Arbeiderspers, Amsterdam

Vos, K./Gründemann, R. (2008): Globalisering en arbeidsverhoudingen, in: Tijdschrift voor Artbeidsvraagstukken 3, S. 309-325

Zugehör, R. (2003): Die Zukunft des Rheinischen Kapitalismus: Unternehmen zwischen Kapitalmarkt und Mitbestimmung, Wiesbaden 